\title{
Demand Response Model for optimized use of Renewable Energies in Production
}

\author{
Clemens Faller ${ }^{1}$, Sonja Podjawerschek ${ }^{1}$, Armin Co ${ }^{1}$, Markus Dannehl ${ }^{1}$, Moritz Paul \\ Heimbach ${ }^{1}$, Sory Ibrahima Nadiaye ${ }^{1,}$ \\ ${ }^{1}$ Bochum University of Applied Sciences, Germany

\begin{abstract}
A demand-response model was developed in the Automation Technology Laboratory at the Velbert/Heiligenhaus Campus $(\mathrm{CVH})$ of Bochum University of Applied Sciences, in which energy users in the manufacturing sector are networked with a smart grid via a cloud platform in order to control production based on the supply of renewable energies.
\end{abstract}

Keywords: Demand-Response, Smart-Grid, Cloud-Platform, IOT, Industry 4.0

\section{Introduction}

Due to the increasing scarcity and thus price increase of energy, companies active in production are already under pressure to increase the resource efficiency of their production facilities. In the interaction between energy producers and users, data exchange enables optimized use of the available energy in order to minimize the reserve to be held from conventional energy sources [1]. As the specific energy consumption is constantly reduced, but at the same time the economy and thus also the manufacturing industry is growing, there is a need for further action with regard to the energy efficiency of production in order to counteract this rebound effect [2]. In order to provide manufacturing SMEs with a basis for analyzing their energy consumption in relation to the product produced and the operating resources from both an energy and productivity point of view, it is necessary to develop a manageable solution [3]. Such a manageable system for a demand response scenario, in which production and consumption of energy is balanced [4] was developed in the Automation Technology Laboratory at the Velbert/Heiligenhaus Campus (CVH) of Bochum University of Applied Sciences, in which energy users in the manufacturing sector are networked with a smart grid via a cloud platform in order to control production based on the supply of renewable energies.

\section{Concept of the Demand-Response Architecture}

The demand response model contains a smart grid model (SGM), a production facility and a cloud platform. The SGM illustrates an intelligent energy network with various electricity generators and consumers. This included both hardware and software components. The group of generators on the SGM is made up of one conventional component (a conventional power plant) and two renewable energy sources, a wind turbine and a photovoltaic farm. The group of consumers is represented by a cluster of houses. All these components are interconnected on the model. The connections are LED strips that can light up both green and red. Green stands for renewable energy and red for conventional energy. The wind turbine on the model has a motor, which can be used to illustrate the wind speed. The LED strips and the wind turbine are controlled by an Arduino, which adjusts the color and rotation speed via PWM. 
The smart factory model is a system from Festo Didactic. This model consists of a conveyor belt and pneumatic actuators. A sorting task is carried out as an example application. The control system is a RevolutionPi (RaspberryPi in an industrial housing) with a CoDeSys PLC Runtime.

Both parts of the demand response model, i.e., the smart grid model and the production system, are connected via OPC-UA to an edge gateway, which sends the energy data to a cloud platform from the company EXOR. The demand-response application then runs on this cloud platform. The following scenarios were developed as part of the work: When a production order is to be started, it is calculated within the cloud platform whether sufficient renewable energies are available. If this is the case, the PLC receives the signal to start the sample application. If not enough renewable energy is produced in the smart grid model, the person responsible for production receives this information and can then decide, based on the importance of his order, to start production anyway or to wait for better availability of renewable energy sources.

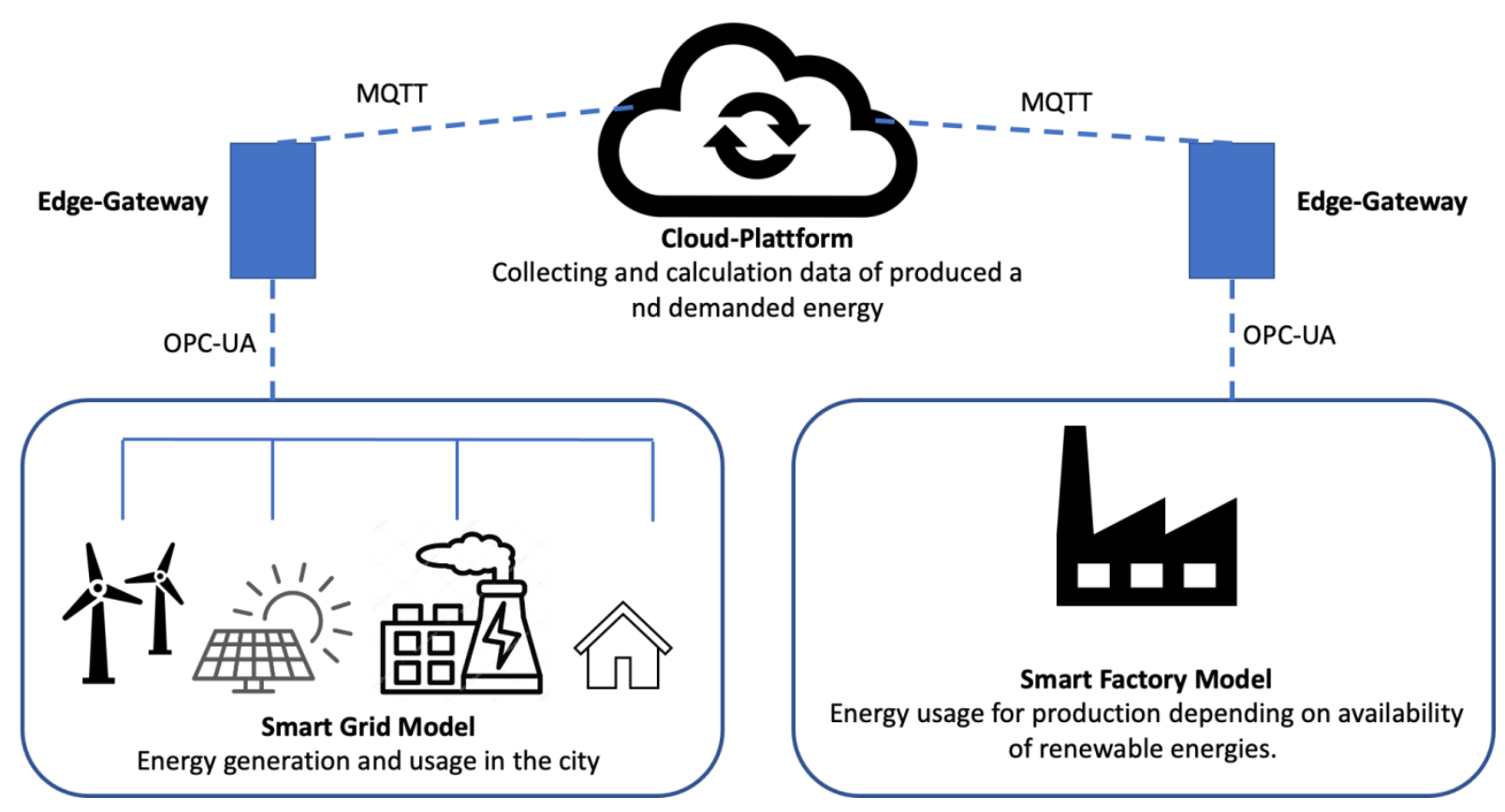

Figure 1. The demand response scenario.

\section{Development of the Smart Grid Model}

The SGM is a model of a city that uses electricity from conventional power plants when the electricity from renewable sources is not sufficient to supply the city. The wind turbine is not a real wind turbine in this sense, since it is driven by a motor and thus does not produce energy. It is only meant to serve as a visual object and simulate the energy generated from wind power. The model of the solar plant consists of models, which are supposed to represent solar panels. A brightness sensor is attached to one of these angles. This is connected to both the Arduino and the Raspberry $\mathrm{Pi}$ via its $\mathrm{I}^{2} \mathrm{C}$ interface and supplied with $3.3 \mathrm{~V}$ voltage. Furthermore, a halogen lamp is mounted on SGM, which can be dimmed by minimizing the voltage supply. This allows to simulate the generation of photovoltaic energy.

On SGM the consumption distribution is displayed by means of LEDs. These LEDs are RGB LEDs, so that they can shine green as well as red. The red LEDs symbolize the coverage of consumers by conventional power generation, and the green LEDs symbolize the coverage by renewable energy sources. 
For the representation of the energy production a simulation was developed, which represents a daily expiry, with time-of-day dependent consumption by dwellings and energy production by the renewable sources described above. Thus, at different times of the day there is sometimes a surplus of renewable energy and sometimes the supply is covered exclusively by conventional energy. Depending on the energy distribution within the grid, the LEDs are switched to visualize the status.

The OPC UA server should make it possible to simulate the simulation state and simultaneously switch consumers in the model on and off. For control purposes, the OPC UA Server therefore provides three control variables and also releases the state of the simulation to the outside:

- sim_mode_enabled This variable of type Boolean can be used to select between simulation and manual control modes.

- time This variable of type int can be used to set a time between 0 and 23 o'clock.

- producing This variable of type Boolean can be used to control whether an additional consumer (e.g., an industrial plant) is to be switched on or off in the model.

- excess_power (Surplus) renewable energy available in the grid.

- power_production Total energy provided, both renewable and conventionally.

- renewable_pwer Renewable energy produced.

- sun_power Energy produced by the photovoltaic park. used_power Commuted energy of all consumers. wind_power Energy produced by the wind turbine.
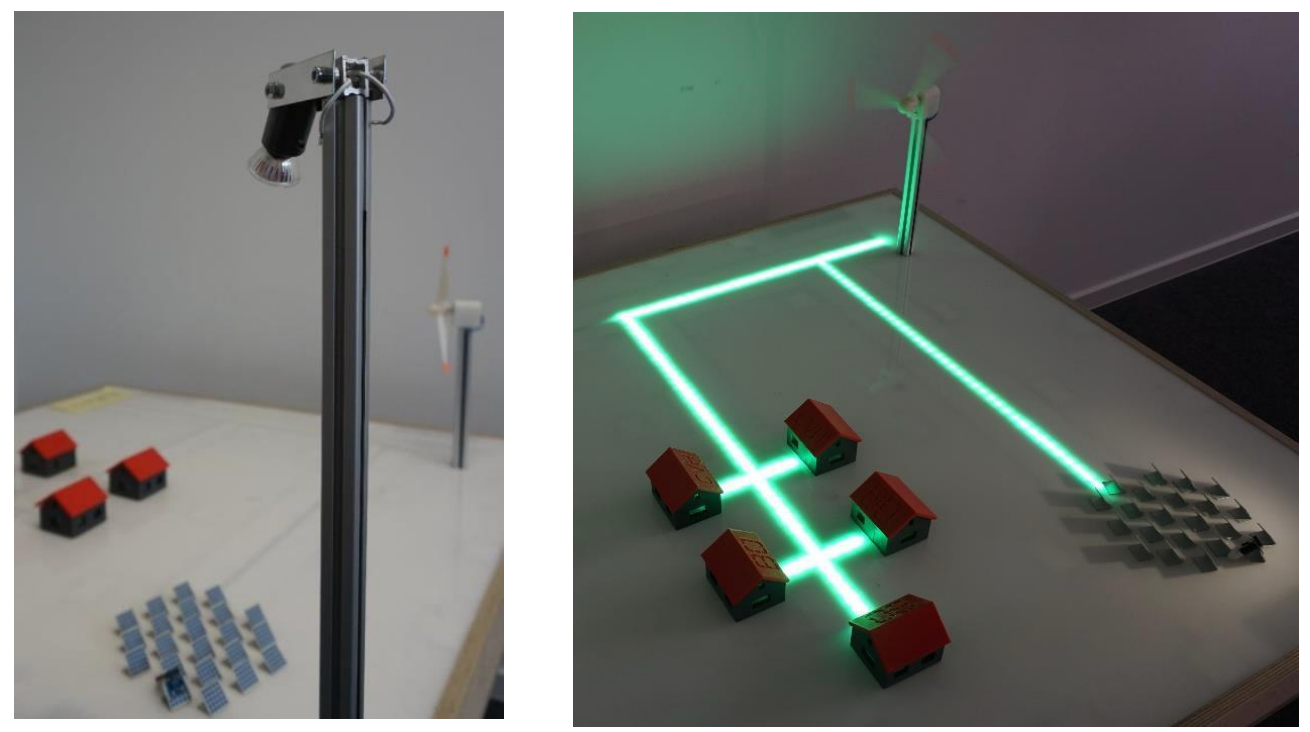

Figure 2. The smart grid model.

\section{Development of the Production Model}

The model of the smart factory to be controlled is an electropneumatic system with actuators and sensors from Festo. The core task of the system is the separation of workpieces with metal cores from workpieces without metal cores.

The core of the system is a conveyor belt that can be moved in both directions at two speeds and is equipped with a light barrier at both ends. At point 1, there is a magazine for workpieces, which is equipped with a light barrier and into which a pneumatic cylinder for pushing out is integrated. At station 2 there is a light barrier, an inductive sensor and a pneumatic cylinder that can pull workpieces down from the assembly line onto a ramp. There is also a light barrier in the ramp. 
The process sequence for a workpiece is as follows: If there is at least one workpiece in the magazine, it is pushed onto the conveyor belt by the pneumatic cylinder. When the workpiece has been completely pushed out by the cylinder, the cylinder retracts, and the conveyor belt moves at the higher of the two speeds in the direction of station 2 . If the light barrier is interrupted at station two, the conveyor belt speed is reduced so that the workpiece is moved slowly along under the inductive sensor. If a metal core is detected in the workpiece, it is pushed into the chute by the pneumatic cylinder and the conveyor belt is then stopped. If the light barrier is released again without a metal core being detected, the workpiece passes through the light barrier at the end of the conveyor, whereupon the conveyor stops.

The start button starts the production of one or more workpieces. If there is not enough renewable energy supplied by the smart grid model, the start button also confirms that you want to manufacture despite the lack of renewable energy. During production, the LED of the start button lights up. By pressing the Stop button, production in automatic mode ends after the current workpiece.

The left lamp Q1 lights up if there is not enough renewable energy. The right lamp Q2 lights up if a start of production has been requested despite insufficient renewable energy and this must be confirmed by a renewed start command.

For the control of the Festo plant a Revolution Pi Core 3 is used, which has a CoDeSys installation as PLC runtime.

The OPC server within the CoDeSys runtime delivers six variables to the edge gateway:

- StartSignalFromCloud has the same effect as pressing the start button on the line. If the variable is set to True while enough renewable energy is available, the production starts, if not enough renewable energy is available, the variable has to be set to False and then again to True to start the production process. (Data type Bool)

- StopSignalFromCloud has the same effect as pressing the stop button on the system. In automatic mode the production is set according to the current workpiece. (Data type Bool)

- NeededPowerInKW denotes the continuous power that the plant consumes in the production process. (Float data type, value 200)

- EnoughRenewableEnergy represents whether the currently available power from renewable energy is greater or less than the power required by the plant. (Data type Bool)

- NumToProduce is the number of parts to be produced in sequence by the plant in automatic mode. For each produced part this variable is reduced by 1. (Data type INT) 
- IsProducing indicates whether the system is currently producing and thus consumes energy. (Data type Bool)

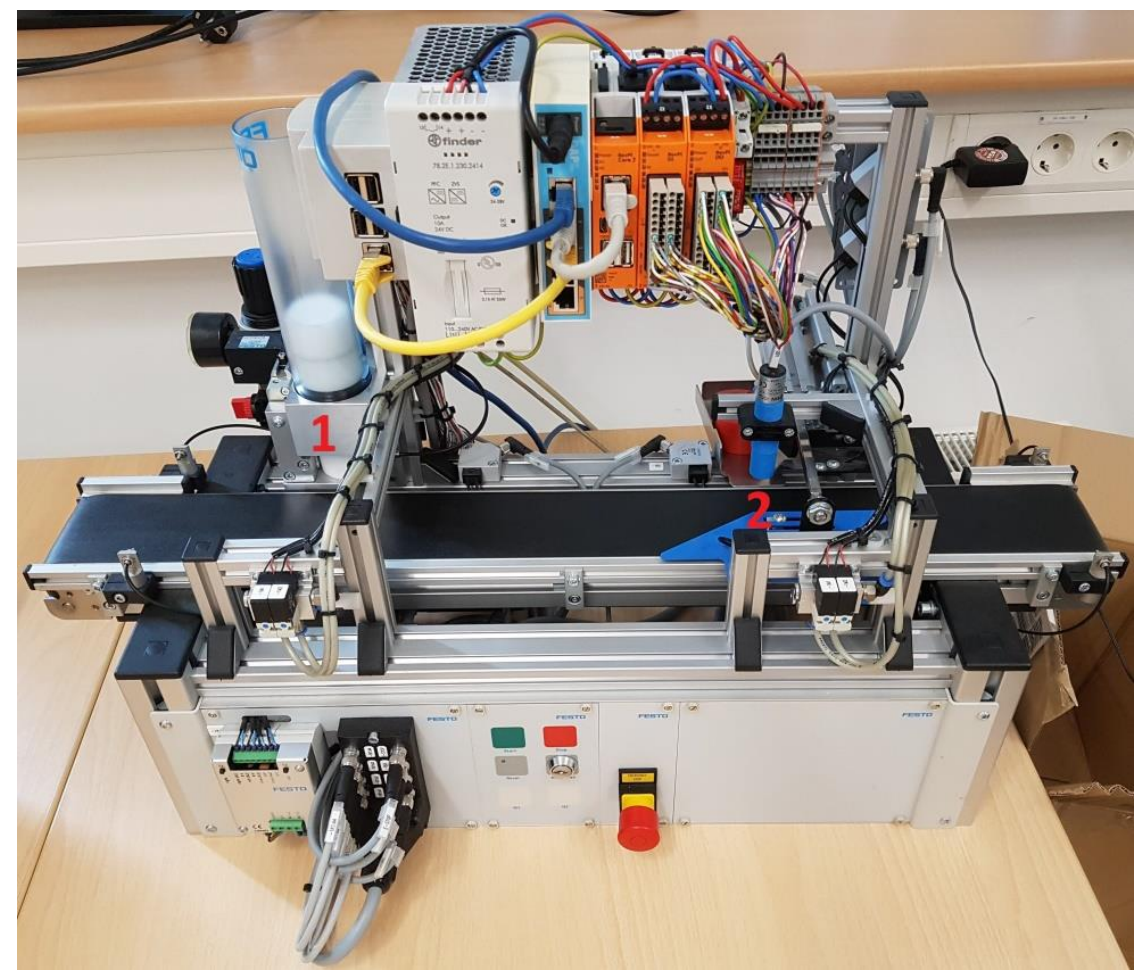

Figure 3. The smart factory model.

\section{Implementation of the Cloud-Solution}

The energy data from the smart grid and they will be connected via an OPC UA connection to an edge gateway, which will evaluate the relevant data and make it available on the cloud platform via MQTT. Within the cloud platform, the corresponding calculations for the demand response scenario must be performed. In the specific use case, the required power of the production plant should be compared with the given power of the smart grid plant for this purpose. As long as the smart grid provides enough power from renewable sources for production, the variable "EnoughRenewableEnergy" in the production plant should be set to "TRUE". For this purpose, a graphical view is created, and a number field is inserted in it via the "Widget Gallery". The number field is first connected to the variable "Excess_Power" To execute the calculation on every data change, an "OnDataUpdate" event is created, which triggers a JavaScript.

Now these variables can be assigned to the tags of the gateway by mapping. To do this, a tag list of the created device can be opened in the web interface. Then the tags can be assigned from the tag list to the variables in the mapping using drag-and-drop. Various settings can then be made, e.g. whether the variables are only available for read access or also for write access. Once all tags have been assigned, the mapping can be saved.

The variables loaded into the cloud and calculated there can be visualized under "Dashboards" within the cloud platform. Various layouts can be specified here via the "Gallery", which can then be filled with text and number fields, for example. Either fixed values can be assigned to the individual elements or a link can be created with the tags. A link can be created by dragging and dropping the tags from the "Devices" list into the "Value" field of the respective element. Once all settings are complete, the dashboard can be saved. It can then be found via the "Dashboard" menu item and viewed with live values at any time via the "Eyes" icon. 


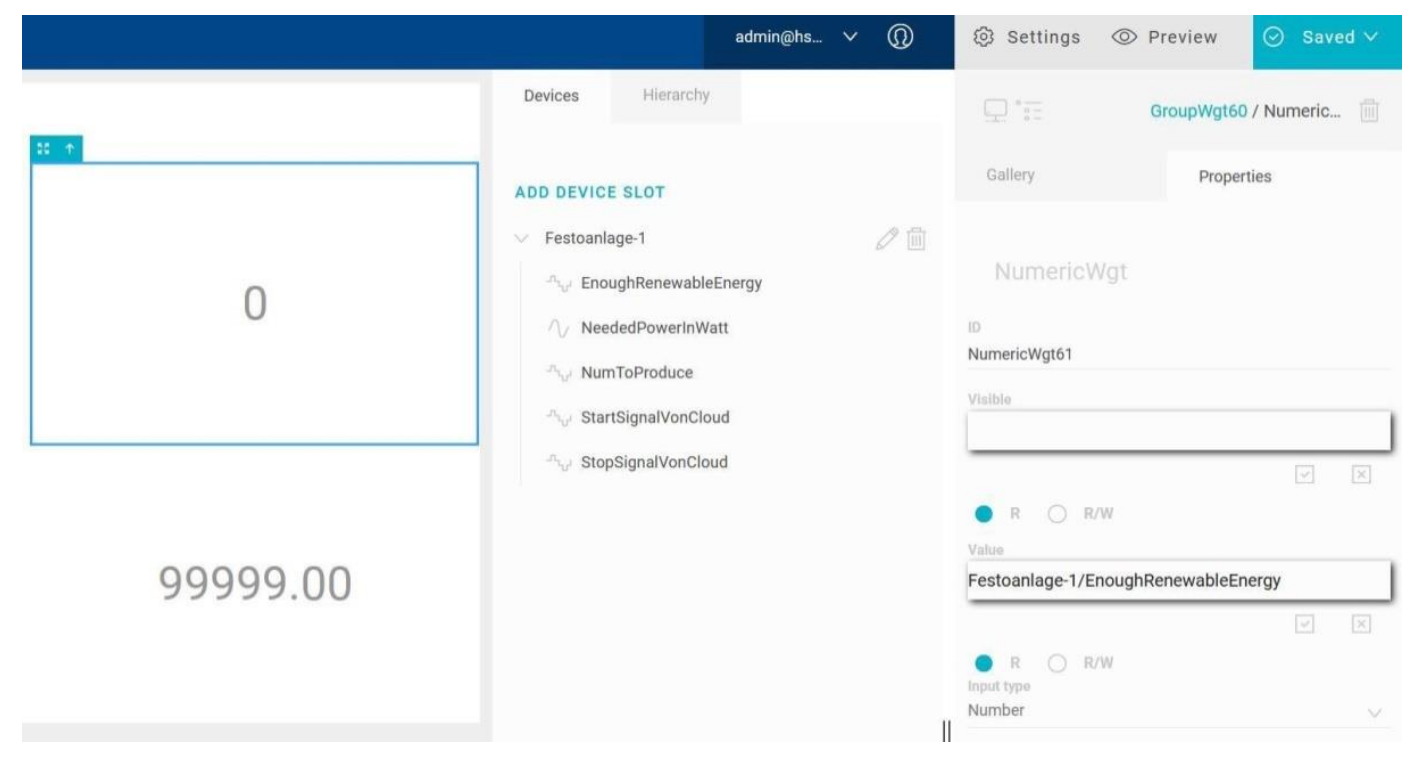

Figure 4. Web interface of the cloud platform.

\section{Conclusion}

The developed model shows a simple implementation of a demand response scenario to adapt the energy demand of a production to the availability of renewable energy. Standard technologies like CoDeSys PLC, OPC-UA and MQTT as cloud connection provide simple and manageable solutions to overcome the silo boundaries between energy production and consumption.

\section{References}

[1] Klima und Energie. http://www.hightech-strategie.de/de/83.php. Accessed 2018 December 18.

[2] Neugebauer R, Westkämper E, Klocke F, Kuhn A, Schenk M, Michaelis A, Spath D, Weidner E. Energieeffizienz in der Produktion. Untersuchung zum Handlungs- und Forschungsbedarf. München: Fraunhofer Gesellschaft; 2008.

[3] Industrie 4.0. http://www.hightech-strategie.de/de/59.php. Accessed 2018 December 6.

[4] Du P, Lu N, Zhong H. Demand Response in Smart Grids. 1. Springer International Publishing; 2019. https://doi.org/10.1007/978-3-030-19769-8 